\title{
Assessment of 18F-FDG uptake in idiopathic pulmonary fibrosis: influence of lung density changes
}

\author{
A. Castiaux ${ }^{1 *}\left(\mathbb{D}, \mathrm{G}\right.$. Van Simaeys ${ }^{1}$, S. Goldman ${ }^{1}$ and B. Bondue ${ }^{2^{*}}$
}

\author{
* Correspondence: casamelie@ \\ hotmail.com; bbondue@ulb.ac.be \\ ${ }^{1}$ Department of Nuclear Medicine, \\ Erasme Hospital, Université Libre de \\ Bruxelles, route de Lennik 808, \\ B-1070 Brussels, Belgium \\ ${ }^{2}$ Department of Respiratory \\ Medicine, Erasme Hospital, \\ Université Libre de Bruxelles, route \\ de Lennik 808, B-1070 Brussels, \\ Belgium
}

\begin{abstract}
Background: Idiopathic Pulmonary Fibrosis (IPF) is a progressive and irreversible disease leading to terminal respiratory insufficiency. Fluorodeoxyglucose ([18F]-FDG) PET/CT has been proposed to track the activity of the disease. However, IPF is characterized by regional changes in lung density that affects the FDG uptake, a factor generally not taken into account in previous studies. In this work, we studied the relationship between severity of IPF and lung uptake of [18F]-FDG, evaluated by mean and maximum standardized uptake value (SUV), corrected (SUVmean-corr; SUVmax-corr) and uncorrected for lung density (SUVmean-uncorr; SUVmax-uncorr).

Methods: [18F]-FDG PET/CT was performed in 31 IPF patients between 2013 and 2017. Lung density was determined on CT. SUV values were correlated with lung function tests, carbon monoxide diffusion (DLCO) and 6-min walking test (6MWT) at baseline and at 1 year. Correlation with the GAP index, a well-validated prognostic score in IPF, was also determined.
\end{abstract}

Results: At baseline, SUVmean-uncorr was highly correlated with lung density ( $r=0$. 755; $p<0.001)$. SUVmean-uncorr and lung density were correlated with lung function tests (vital capacity (VC): $p=0.013$ and $p=0.003$; forced vital capacity (FVC): $p=0.004$ and $p=0.001$; total lung capacity (TLC): $p=0.001$ and $p=0.001$, respectively), while SUVmean-corr was not (VC: $p=0.733$; FVC: $p=0.667 ; \mathrm{TLC}: p=0$. 382). Interestingly, SUVmean-corr was significantly higher in patients with a GAP index of $3(p=0.005)$, and negatively correlated with DLCO $(r=-0.398 ; p=0.026)$ and desaturation during the $6 \mathrm{MWT}(r=-0.401 ; p=0.024)$. But no correlation was found with changes in lung function tests, walk distance and DLCO at 1 year.

Conclusion: To evaluate the role of ([18F]-FDG) PET/CT in IPF, correction for lung density appears necessary. As suggested by the correlation with DLCO, densitycorrected SUV seems related to the intrinsic disease activity and particularly to the integrity of the alveolar-capillary barrier. However, ([18F]-FDG) PET/CT has probably a limited prognostic value as no correlation was found between SUVmean-corr and the clinical evolution at 1 year. Further studies with a longer follow-up are warranted.

Keywords: PET/CT, Fluorodeoxyglucose, SUV, Idiopathic pulmonary fibrosis, Lung density 


\section{Background}

Idiopathic pulmonary fibrosis (IPF) is a chronic, irreversible, and progressive fibrosing interstitial lung disease leading to a terminal respiratory insufficiency (Raghu et al., 2011). Although the aetiology of IPF remains unknown, it appears that recurrent alveolar epithelial cell injury play a central role with an aberrant healing process characterized by accelerated apoptosis of type II alveolar epithelial cells, abnormal recruitment of fibroblasts (which in turn differentiate to myofibroblasts) and exaggerated production of extracellular matrix components (Spagnolo et al., 2014).

Prognosis of untreated IPF is poor with a median survival time of 2 to 3 years from the time of diagnosis (Raghu et al., 2011). Apart from an ineluctable evolution towards respiratory failure, IPF is associated with an increased incidence of lung cancer (Archontogeorgis et al., 2012). Its clinical course is unpredictable with the majority of patients experiencing a slow progression over many years, while others show an accelerated decline with or without acute exacerbations (Raghu et al., 2011). Identification and validation of predictive markers of the clinical evolution are therefore crucial.

Some prognostic factors are already known such as a decline in forced vital capacity (FVC) or carbon monoxyde diffusion (DLCO) (Raghu et al., 2011). The GAP risk assessment system is a clinical tool estimating the prognosis of patients with IPF. The GAP index is a composite based on gender $(\mathrm{G})$, age (A), and physiological variables (P) (FVC, DLCO). This score predicts the overall risk of mortality of a patient but it does not inform about disease activity at a specific time point and about rate of evolution (Ley et al., 2012; Salisbury et al., 2016).

Value of 2-deoxy-2-(18F)fluoro-D-glucose positron emission tomography with computed tomography ([18F]-FDG PET/CT) has been recently studied in IPF. Several studies showed that IPF patients have increased lung uptake of [18F]-FDG in areas of reticular/ honeycombing and ground-glass opacity on high-resolution CT (HRCT) (Groves et al., 2009) and in areas of normal pulmonary parenchyma (Win et al., 2014). Moreover, [18F]-FDG lung uptake seems to be correlated with the severity of the disease, and to reflect not only the inflammatory process but also fibrogenesis (Groves et al., 2009; Meissner et al., 2006). This is supported by a study in a murine model of pulmonary fibrosis showing [18F]-FDG uptake during the initial inflammatory phase of the bleomycin-induced lung injury but also in the latter fibrotic phase (Bondue et al., 2015).

Two recent studies evaluated the prognostic value of the standardized uptake value (SUV) in IPF patients (Lee et al., 2014; Justet et al., 2017). The mean lung SUV (SUVmean) was negatively correlated with FVC and DLCO (Lee et al., 2014; Justet et al., 2017), and the decline in FVC was associated with an increment in the maximum lung SUV (SUVmax) (Lee et al., 2014). This suggests that [18F]-FDG uptake is related to the functional impairment and clinical severity in IPF.

However, the prognostic value of the [18F]-FDG PET/CT in IPF could be explained - at least partially - by a relationship with lung density, depending on the way the pulmonary compartment is considered for the quantification of [18F]-FDG uptake, i.e. including or excluding the air volume from the lung volume of interest. Indeed, IPF lungs are smaller, with a thickened interstitium resulting in a higher tissue/air ratio compared to normal lung. Even if tissue uptake remains constant, a higher tissue/air ratio results in higher SUV values, when the whole lung volume is used to quantify [18F]-FDG uptake in the pulmonary tissue. Pulmonary function impairment evolves in 
parallel with lung density, so any relationship between pulmonary function and [18F]-FDG uptake in an undifferentiated lung volume may be driven by density changes, regardless of the metabolic demands by the pulmonary cells involved in inflammatory or fibrotic processes. So, if [18F]-FDG PET/CT is used to assess the intrinsic activity of the disease (and not only its severity), the influence of lung density should be taken into consideration in the [18F]-FDG uptake evaluation (Lambrou et al., 2011; Holman et al., 2015).

The aim of this study was to correlate the severity and the evolution of IPF to lung uptake of [18F]-FDG, evaluated by SUVmean and SUVmax, corrected (SUVmean-corr; SUVmax-corr) and uncorrected (SUVmean-uncorr; SUVmax-uncorr) for lung density. We hypothesize that [18F]-FDG lung uptake corrected for lung density can be a prognostic factor of the pulmonary function decline.

\section{Methods}

Patients

A total of 31 IPF patients ( 23 men, 8 women) were included in the study. These patients were followed at the Erasme hospital until April 2018 and a [18F]-FDG PET/CT scan was performed between 2013 and 2016. The diagnosis of IPF was established in accordance with the ATS/ERS/JRS/ALAT recommendations (Raghu et al., 2011) through a multidisciplinary discussion involving pulmonologists, radiologists, internal medicine specialists (or rheumatologists) and pathologists experienced in the diagnosis of interstitial lung diseases (ILD). The patients were not treated by antifibrotic drugs at the time of the PET/ $\mathrm{CT}$, but most of them receive such a treatment during the follow-up period (pirfenidone, $n=15$; nintedanib, $n=15$; no treatment, $n=1$ ). Patients under immunosuppressive therapy were excluded, with the exception of those receiving a low dose of corticosteroids (4 mg or less of methylprednisolone or equivalent). The protocol has been approved by the Erasme hospital Ethics Committee (ref. P2016/427; EudraCT/CCB: NA). Written informed consent for participation in the study was obtained from all the patients.

\section{Clinical investigations}

Information obtained included age, sex, tobacco exposure and treatments. For each patient, we analysed the pulmonary function tests (PFT) at the time of PET/CT and then every 3 months for at least 1 year of follow-up after the PET/CT evaluation. The following parameters were recorded: diffusing capacity of the lung for carbon monoxide (DLCO), forced vital capacity (FVC), vital capacity (VC) and total lung capacity (TLC). A 6-min-walk test (6MWT) was performed at the time of PET/CT and 1 year later. For each patient, the GAP index was calculated as described by Ley and al. (Ley et al., 2012). When available, differential cell counts from bronchoalveolar lavage (BAL) were recorded if BAL was performed 24 months or less before PET/CT (BAL performed during suspected infection or acute exacerbation were excluded).

To assess the clinical evolution of the patients at 1 year, the slope of the evolution of the VC, FVC, TLC, and DLCO were determined with a linear regression using least-squares method, in order to cope with the variability of PFT measurements performed routinely in clinical practice. The results of the 6MWT performed at the time of the PET/CT and at 1 year were also recorded. The difference between the pre- and 
post-exercise oxygen saturation $\left(\mathrm{SaO}_{2}\right)$ at the time of PET/CT was calculated as well as the change in $\mathrm{SaO}_{2}$ post-exercise and the difference in walking distance at 1 year.

The disease activity for each patient was further characterized as "progressive" or "non-progressive". A patient is categorized with a progressive disease if any of the following change occurred during the follow-up period of 1 year: $\geq 10 \%$ relative decrease from baseline in FVC, $\geq 15 \%$ relative decrease from baseline in DLCO, $\geq 5 \%$ decrease in the $\mathrm{SaO}_{2}$ after exercise at 6MWT, lung transplantation or death related to IPF.

\section{PET-CT imaging}

All imaging examinations were performed using a dedicated PET/CT scanner (Gemini GS16P, Philips Medical Systems, Cleveland, OH, USA). All patients were fasted for at least $6 \mathrm{~h}$ before the examination. Blood glucose level before [18F]-FDG injection had to be lower than $150 \mathrm{mg} / \mathrm{dL}$. PET-CT images were obtained $60 \mathrm{~min}$ after injection of 300-370 MBq of [18F]-FDG. Low-dose CT was used for attenuation correction of the PET emission data. PET/CT was performed outside of an acute exacerbation. Acute exacerbation is defined by the sudden onset (within a month) of dyspnoea, associated with the appearance of new ground glass images on the chest CT, not fully explained by cardiac failure or fluid overload, and without alternative diagnosis such as pneumothorax, pleural effusion or pulmonary embolism (Collard et al., 2016).

Three-dimensional regions of interest were drawn on pulmonary parenchyma in the left and right lung using the Vivoquant software. In these regions, the SUVmean and SUVmax were obtained and corrected for the tissue fraction, according to a method already validated (Lambrou et al., 2011; Holman et al., 2015). Briefly, CT images were obtained for each patient to determine the lung density in Hounsfield unit $\left(\mathrm{HU}_{\text {Lung }}\right)$. Then, a coefficient $\mathrm{k}$ corresponding to the tissue fraction was calculated as follow and as previously published (Lambrou et al., 2011):

$$
\mathrm{k}=\frac{\mathrm{HU}_{\text {Lung }}-\mathrm{HU}_{\text {Air }}}{\mathrm{HU}_{\text {Tissue }}-\mathrm{HU}_{\text {Air }}}
$$

Where $\mathrm{HU}_{\text {Lung, }}, \mathrm{HU}_{\text {Tissue }}$ and $\mathrm{HU}_{\mathrm{Air}}$ are the densities (in Hounsfield units) for the lung, soft tissues and air, respectively. $\mathrm{HU}_{\text {Lung }}$ is determined on the $\mathrm{CT}, \mathrm{HU}_{\text {Tissue }}$ is estimated equal to 50, and $\mathrm{HU}_{\text {Air }}$ equals $-1000 \mathrm{HU}$. A matrix containing all these tissue fractions can be created, allowing the PET images to be corrected by dividing the SUV values in each voxel by the corresponding tissue fraction.

$$
\mathrm{SUV}_{\text {corr }}=\frac{\mathrm{SUV}_{\mathrm{uncorr}}}{\mathrm{k}}
$$

\section{Statistical analysis}

Correlations between imaging and clinical variables were analysed using Pearson correlation tests. Multiple comparisons were performed using ANOVA tests with Bonferroni post hoc analysis. According to the results of the D'Agostino \& Pearson test used to assess the normality of the sample values, simple comparisons between two groups were tested by unpaired Student $\mathrm{t}$ tests or Mann-Whitney tests. Statistical analyses were performed using the GraphPad Prism 7.03 software. For all tests, a $p$-value of less than 0.05 was considered statistically significant. 


\section{Results}

Between 2013 and 2016, 31 IPF patients were included in the study. Their clinical characteristics at the time of the inclusion are summarized in Table 1. Ten patients died during the study period, and three patients underwent lung transplantation. A total of 29 patients reached a follow-up of 1 year after the PET/CT and were classified as having a progressive $(n=15)$ or a non-progressive $(n=14)$ disease. Two patients were

Table 1 Clinical and functional data for patients at baseline

\begin{tabular}{|c|c|c|c|c|}
\hline Characteristics & $\begin{array}{l}\text { Patients } \\
(n=31)\end{array}$ & $\begin{array}{l}\text { Progressive } \\
(n=15)\end{array}$ & $\begin{array}{l}\text { Non-progressive } \\
(n=14)\end{array}$ & $p$ value \\
\hline Age (years, mean $\pm S D$ ) & $69.8 \pm 8.6$ & $70.0 \pm 9.0$ & $68.6 \pm 8.6$ & $p=\mathrm{NS}$ \\
\hline Sex ratio (M/F) (n) & $23 / 8$ & $12 / 3$ & $9 / 5$ & $p=\mathrm{NS}$ \\
\hline \multicolumn{5}{|l|}{ Smoking status (n) } \\
\hline Never smoker & 4 & 2 & 2 & \\
\hline Current smoker & 0 & 0 & 0 & \\
\hline Former smoker & 25 & 12 & 11 & \\
\hline Passive smoker & 2 & 1 & 1 & \\
\hline \multicolumn{5}{|l|}{ Treatments (n) } \\
\hline Pirfenidone & 15 & 7 & 7 & \\
\hline Nintedanib & 15 & 8 & 6 & \\
\hline No treatments & 1 & 0 & 1 & \\
\hline \multicolumn{5}{|l|}{ GAP score (n) } \\
\hline Stage 1 & 10 & 2 & 8 & \\
\hline Stage 2 & 16 & 8 & 6 & \\
\hline Stage 3 & 5 & 5 & 0 & \\
\hline C-reactive protein (mg/L) (median, range) & $3.2(1.9-7)$ & $2.7(1.7-5.6)$ & $3.75(1.72-8.2)$ & $p=\mathrm{NS}$ \\
\hline \multicolumn{5}{|l|}{ Pulmonary function test (mean \pm SD): } \\
\hline FVC (\% predicted) & $73.9 \pm 19.0$ & $64.4 \pm 13.5$ & $83.8 \pm 19.9$ & $p=0.005 *$ \\
\hline DLCO (\% predicted) & $44.3 \pm 11.7$ & $41.5 \pm 14.4$ & $47.1 \pm 8.3$ & $p=\mathrm{NS}$ \\
\hline \multicolumn{5}{|l|}{$6 \mathrm{MWT}(n=28)($ mean $\pm \mathrm{SD})$} \\
\hline $\mathrm{SaO}_{2}$ post-exercise (\%) & $89.1 \pm 4.7$ & $87.3 \pm 4.0$ & $90.9 \pm 4.2$ & $p=0.035 *$ \\
\hline Distance traveled $(\mathrm{m})$ & $464.4 \pm 111.7$ & $464.8 \pm 118.4$ & $460.4 \pm 114.8$ & $p=N S$ \\
\hline \multicolumn{5}{|c|}{ Bronchoalveolar lavage cytology ( $n=24)$ (median, range) } \\
\hline Cellularity $\left(/ \mathrm{mm}^{3}\right)$ & $293(140-450)$ & $405(130-567.5)$ & $261.5(132.5-420)$ & $p=\mathrm{NS}$ \\
\hline Macrophages (\%) & $79(65-89)$ & $85.5(60.2-92)$ & $79(65-85)$ & $p=\mathrm{NS}$ \\
\hline Lymphocytes (\%) & $4(2.5-7)$ & $3.5(1.75-7)$ & $5.5(2.5-10)$ & $p=\mathrm{NS}$ \\
\hline Neutrophils (\%) & $8(3-15)$ & $6(3.75-10)$ & $8(2-16)$ & $p=\mathrm{NS}$ \\
\hline Eosinophils (\%) & $1(0-3)$ & $1(0-9.5)$ & $2(0-3)$ & $p=\mathrm{NS}$ \\
\hline \multicolumn{5}{|l|}{$\mathrm{PET} / \mathrm{CT}$ parameters (mean $\pm \mathrm{SD}$ ) } \\
\hline SUVmean uncorr & $0.95 \pm 0.19$ & $1.00 \pm 0.20$ & $0.89 \pm 0.17$ & $p=N S$ \\
\hline SUVmax uncorr & $3.24 \pm 0.73$ & $3.34 \pm 0.92$ & $3.12 \pm 0.52$ & $p=N S$ \\
\hline SUVmean corr & $2.70 \pm 0.35$ & $2.72 \pm 0.43$ & $2.65 \pm 0.29$ & $p=\mathrm{NS}$ \\
\hline SUVmax corr & $29.89 \pm 6.43$ & $30.20 \pm 6.74$ & $30.48 \pm 6.26$ & $p=N S$ \\
\hline $\mathrm{CT}$ mean $(\mathrm{HU})($ mean $\pm \mathrm{SD})$ & $-580.04 \pm 57.34$ & $-563.09 \pm 51.78$ & $-598.49 \pm 61.51$ & $p=N S$ \\
\hline
\end{tabular}

$p$ is the value of unpaired Student t tests or Mann-Whitney test for simple comparisons between two groups (progressive vs. non-progressive disease)

NS not significant, SD standard deviation, FVC Forced vital capacity, DLCO carbon monoxide diffusion, 6MWT 6-min walking test, $\mathrm{SaO}_{2}$ oxygen saturation, SUV standardized uptake value 
lost to follow-up. A [18F]-FDG PET/CT was performed at baseline (Fig. 1). The SUVmean and the SUVmax, corrected or not for the tissue fraction, were determined.

The PET/CT analysis at baseline showed a SUVmean-uncorr of $0.95 \pm 0.19$ (mean \pm $\mathrm{SD}$ ), which was strongly correlated with the lung density assessed by the chest CT (CTmean: $-580.04 \pm 57.34 \mathrm{HU}$, mean $\pm \mathrm{SD})(r=0.755$; $p<0.001)$ (Fig. 2). Conversely, when correcting the SUVmean for the tissue fraction (SUVmean-corr), this correlation with the lung density completely disappeared $(r=-0.091 ; p=0.627)$.

Other strong correlations were observed between the lung volumes (FVC, TLC) measured at baseline and the SUVmean-uncorr (FVC: $p=0.004$; TLC: $p=0.001$ ) and the lung density (CTmean) (FVC: $p=0.001$; TLC: $p=0.001$ ) (Fig. 3a and b). Once again, these correlations disappeared when correcting for the tissue fraction (SUVmean-corr) (Fig. 3c).

Of note, no significant correlation was found between the SUV values and the cytological analysis of the BAL (Additional file 1: Table S1).

Using the SUVmean-corr as a PET marker independent of the influence of the lung density, we demonstrated correlations between the SUVmean-corr and the DLCO on one side $(r=-0.399 ; p=0.026)$ and the oxygen saturation during the 6MWT (post-exercise value and changes pre- and post-exercise) on the other side ( $r=-0.376$ and $r=0.402 ; p=$ 0.049 and $p=0.034$, respectively). Interestingly, these correlations were not present for the SUVmean-uncorr or SUVmax corrected or not for lung density (Tab. 2 and Fig. 4a-c).

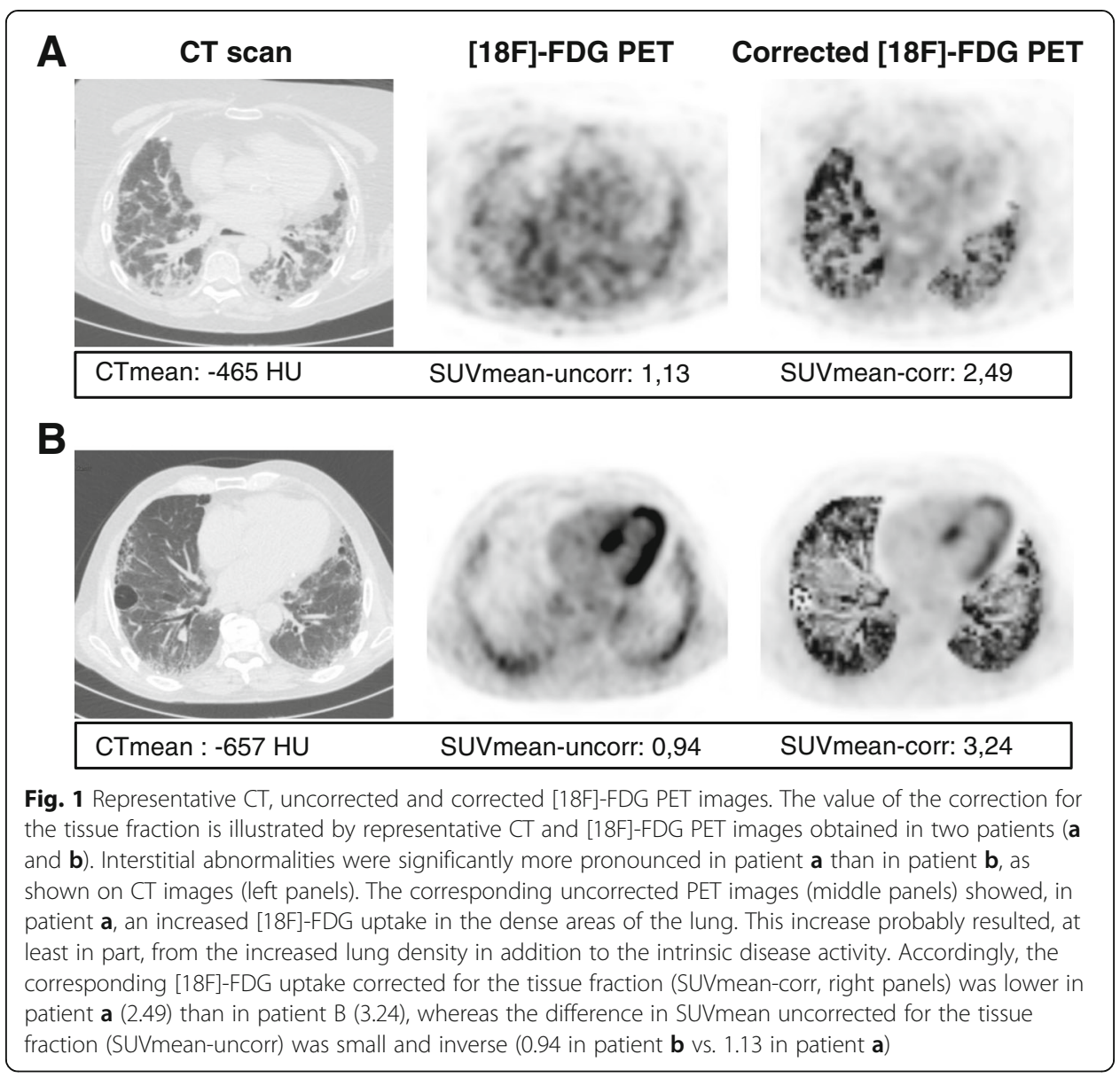



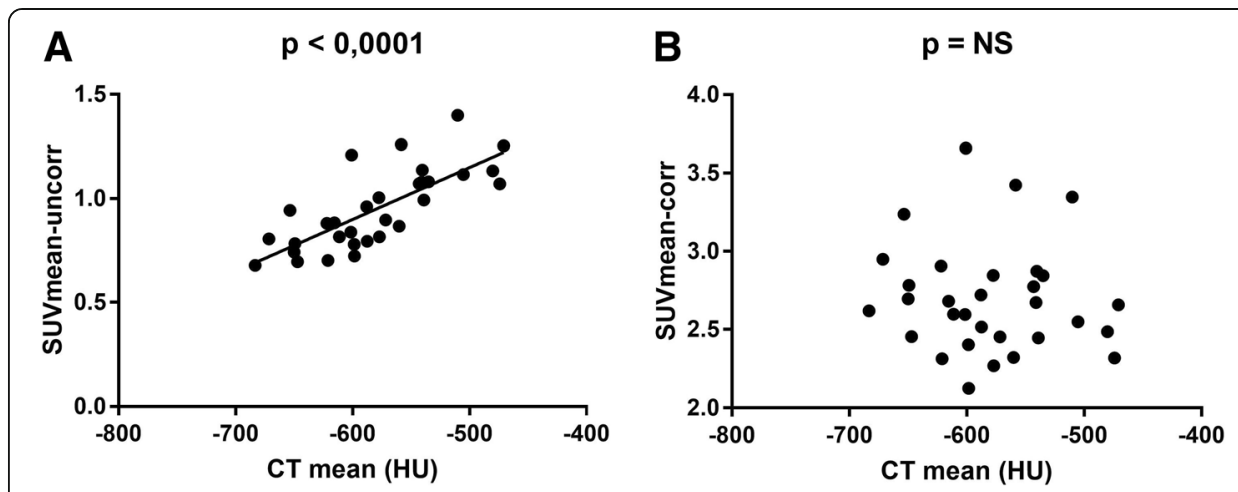

Fig. 2 Correlation between SUVmean and the lung density. The SUVmean (SUVmean-uncorr) (a) and the SUVmean corrected for the tissue fraction (SUVmean-corr) (b) were calculated and correlated to the mean density assessed by the $C T$ scan (CTmean). $n=31$. HU = Hounsfield Unit. NS= not significant
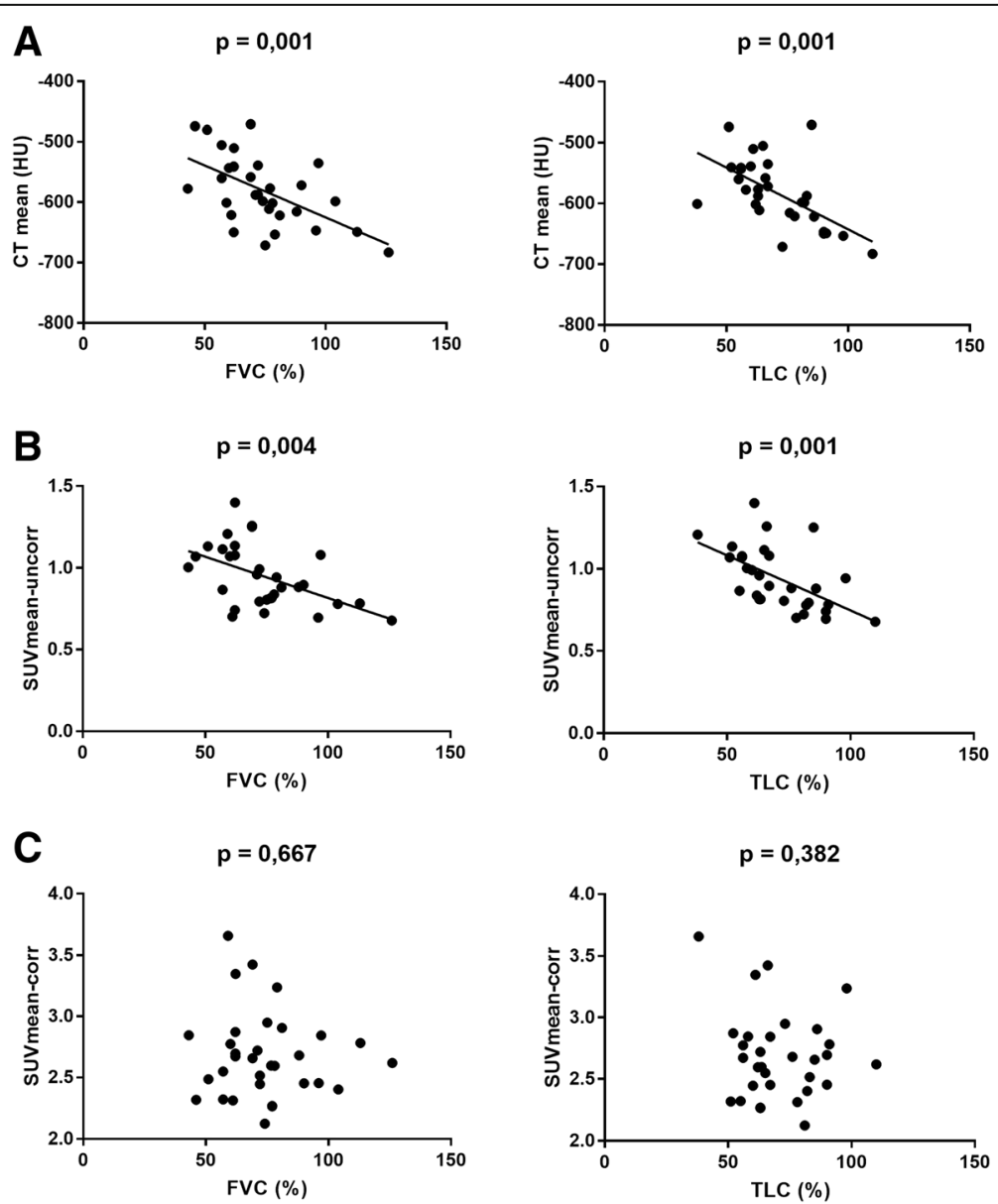

Fig. 3 Correlation between SUV values or the lung density (CTmean) and the lung volumes (FVC, TLC). The CTmean (a) and the SUVmean-uncorr (b) are significantly correlated with lung volumes, while the SUVmean-corr (c) is not. FVC = Forced vital capacity. TLC = Total lung capacity 
Table 2 Main correlations between PET/CT parameters and functional or exercise-related parameters

\begin{tabular}{|c|c|c|c|c|c|c|c|}
\hline \multicolumn{3}{|c|}{ Pearson correlation tests } & \multirow{2}{*}{$\begin{array}{l}\text { SUVmean- } \\
\text { uncorr }\end{array}$} & \multirow{2}{*}{$\begin{array}{l}\text { SUVmean- } \\
\text { corr }\end{array}$} & \multirow{2}{*}{$\begin{array}{l}\text { SUVmax- } \\
\text { uncorr } \\
-0.408\end{array}$} & \multirow{2}{*}{$\begin{array}{l}\text { SuVmax- } \\
\text { corr } \\
-0.082\end{array}$} & \multirow{2}{*}{$\begin{array}{l}\begin{array}{l}\text { CTmean } \\
(\mathrm{HU})\end{array} \\
-0.518\end{array}$} \\
\hline PFT & VC (\%) & $r$ & & & & & \\
\hline & & $p$ & $0.013^{*}$ & 0.733 & $0.025^{*}$ & 0.666 & $0.003^{*}$ \\
\hline & FVC (\%) & r & -0.505 & -0.08 & -0.413 & -0.072 & -0.57 \\
\hline & & $p$ & $0.004^{*}$ & 0.667 & $0.021^{*}$ & 0.7 & $0.001 *$ \\
\hline & TLC (\%) & r & -0.568 & -0.165 & -0.527 & -0.003 & -0.595 \\
\hline & & $p$ & $0.001 *$ & 0.382 & $0.003^{*}$ & 0.987 & $0.001^{*}$ \\
\hline & $\mathrm{DLCO}(\mathrm{ml} / \mathrm{min} / \mathrm{mmHg})$ & r & -0.247 & -0.398 & -0.056 & -0.268 & 0.013 \\
\hline & & p & 0.18 & $0.026^{*}$ & 0.764 & 0.145 & 0.945 \\
\hline \multirow[t]{12}{*}{ 6MWT } & $\mathrm{SaO}_{2}$ post-exercise & r & -0.344 & -0.376 & -0.064 & -0.062 & -0.18 \\
\hline & & $p$ & 0.073 & $0.049^{*}$ & 0.746 & 0.755 & 0.36 \\
\hline & $\Delta \mathrm{SaO}_{2}$ (between the & r & 0.366 & 0.401 & 0.056 & 0.172 & 0.174 \\
\hline & pre- and post-exercise) & $p$ & 0.055 & $0.034^{*}$ & 0.777 & 0.38 & 0.375 \\
\hline & $\Delta \mathrm{SaO}_{2}$ post-exercise (between & r & 0.38 & 0.527 & 0.017 & 0.261 & 0.074 \\
\hline & $\begin{array}{l}\text { the test at the time of PEI and } \\
\text { the test at } 1 \text { year) }\end{array}$ & $p$ & 0.12 & $0.024^{*}$ & 0.947 & 0.296 & 0.769 \\
\hline & Distance traveled & r & 0.037 & -0.217 & 0.354 & 0.032 & 0.199 \\
\hline & & $p$ & 0.85 & 0.266 & 0.064 & 0.87 & 0.311 \\
\hline & SUV mean-uncorr & r & & & & & 0.755 \\
\hline & & $p$ & & & & & $<0.001 *$ \\
\hline & SUV mean-corr & r & & & & & -0.091 \\
\hline & & $p$ & & & & & 0.627 \\
\hline
\end{tabular}

Data presented were obtained using Pearson correlation tests with significant correlations highlighted in bold (corresponding to $p$ values $<0.05$ ). The $r$ corresponds to the correlation coefficient. PFT = pulmonary function tests CV vital capacity, FVC Forced vital capacity, TLC Total lung capacity, DLCO carbon monoxide diffusion, 6MWT 6-min walking test, $\mathrm{SaO}_{2}$ oxygen saturation, SUV standardized uptake value

The patients were also classified according to the GAP score. There was no significant difference in SUVmax-corr between patients in various GAP stages (Additional file 2: Figure S1; $p=0.271$ ), whereas for what concerns SUVmean-corr a significant difference was noticed between patients in stage 3 and in stage $2(p=0.027)$ and between patients in stage 3 and in stage $1(p=0.037)$ (Fig. $4 \mathrm{~d})$. The average SUVmean-corr for stage 1 was $2.62 \pm 0.23$, for stage 2 was $2.62 \pm 0.33$ and for stage 3 was $3.08 \pm 0.43$.

Next, hypothesizing that SUVmean-corr could reflect the intrinsic activity of the disease, we assessed whether it has some predictive value on the clinical outcomes after 1 year of follow-up. A significant correlation was only found between SUVmean-corr and the increase in oxygen desaturation after the 6MWT, assessed by the difference in oxygen saturation at the end of the 6MWT between baseline values and those measured at 1 year (Additional file 3: Figure S2). No significant correlation was found between SUVmean-corr values and changes in lung volumes, walk distance and DLCO at 1 year (Additional file 1: Table S1). When patients with a progressive or a non-progressive disease were compared, no significant difference was found for SUVmean-uncorr $(p=0.124)$, SUVmean-corr $(p=0.626)$, SUVmax-uncorr $(p=0.434)$ and SUVmax-corr $(p=0.909)$ (Fig. 5a and Table 1). Finally, we divided patients according to their vital status at the end of the study (alive vs. death or lung transplantation). Once again, no difference was found for SUVmean-uncorr $(p=0.794)$, SUVmean-corr $(p=0.979)$, SUVmax-uncorr $(p=0.8)$ and SUVmax-corr $(p=0.961)$ (Fig. 5b). 


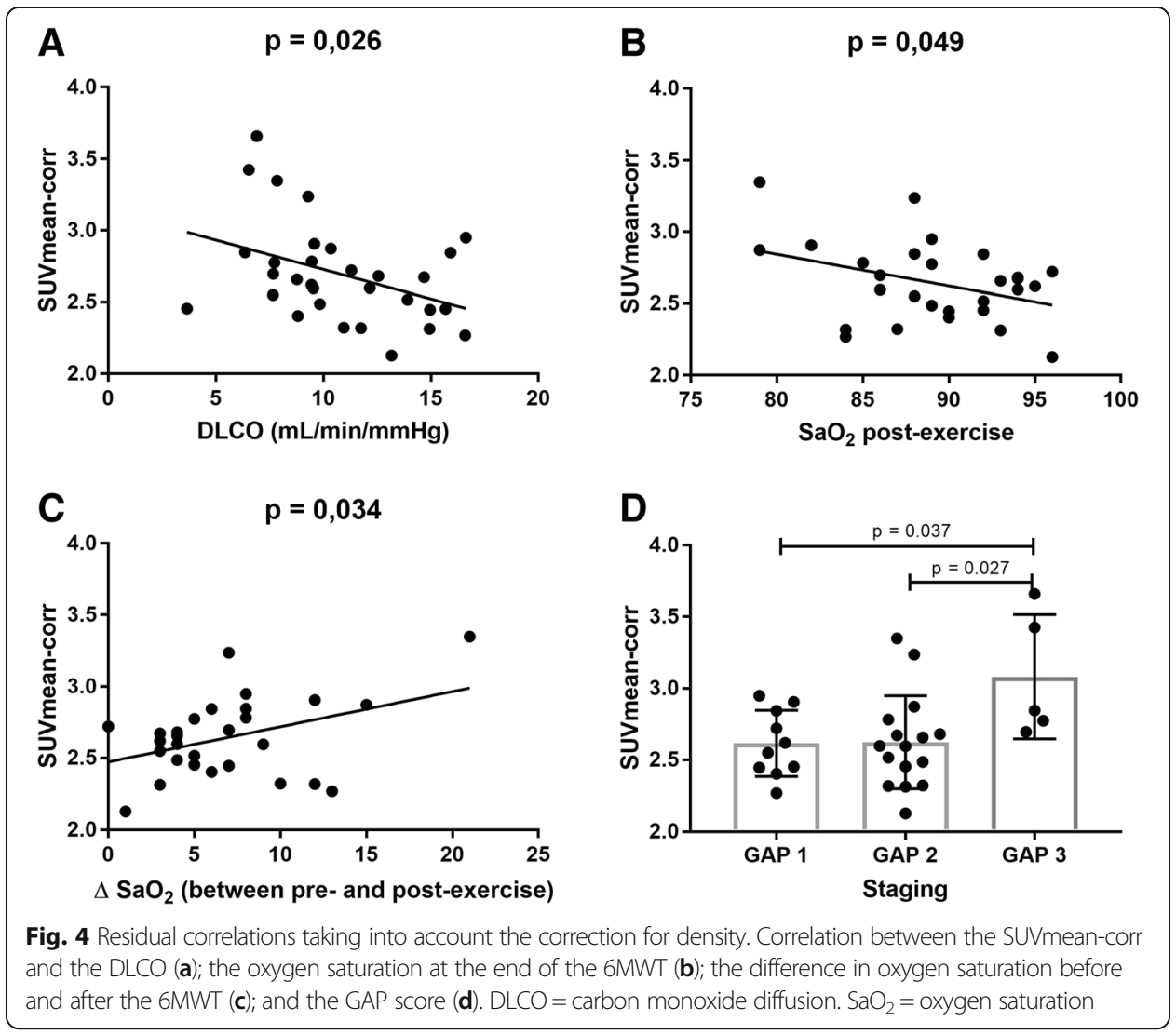

\section{Discussion}

The aim of this study was to evaluate the prognostic value of [18F]-FDG PET/CT in IPF patients. Contrary to previous studies evaluating the usefulness of [18F]-FDG PET/CT in IPF (Meissner et al., 2006; Lee et al., 2014; Justet et al., 2017; Umeda et al., 2015; Win et al., 2018; Nobashi et al., 2016), SUV and derived parameters were corrected for lung density in the present study. We hypothesized that correction for lung density is important since lung density increases with the development of fibrotic tissue, leading to reduced lung volumes and to higher tissue/air ratios compared to normal lung. Even if tissue uptake remains constant, a higher tissue/air ratio will result in higher SUV values. Therefore, if [18F]-FDG PET/CT is used to assess the intrinsic activity of the disease (and not only its severity), the influence of lung density should be taken into consideration in the [18F]-FDG uptake evaluation (Lambrou et al., 2011; Holman et al., 2015).

As expected, mean pulmonary density, as well as uncorrected SUVmean and SUVmax, were negatively correlated with lung volumes (VC, FVC, TLC) measured at the time of PET/CT. This is easily explained by the fact that with the development of fibrotic tissue, density increases while pulmonary compliance decreases, resulting in a reduction in lung volumes. These results are consistent with a pilot study showing that SUVmean uncorrected for density is negatively correlated with FVC (Lee et al., 2014) and other studies in which CTmean values were correlated with pulmonary physiological variables (FVC, TLC) (Ohkubo et al., 2016; Best et al., 2003). SUV corrected for the tissue fraction are not correlated with pulmonary volumes, probably because the volumes are strongly related to density, leaving no residual variability when this primary effect is corrected for. Normalization 

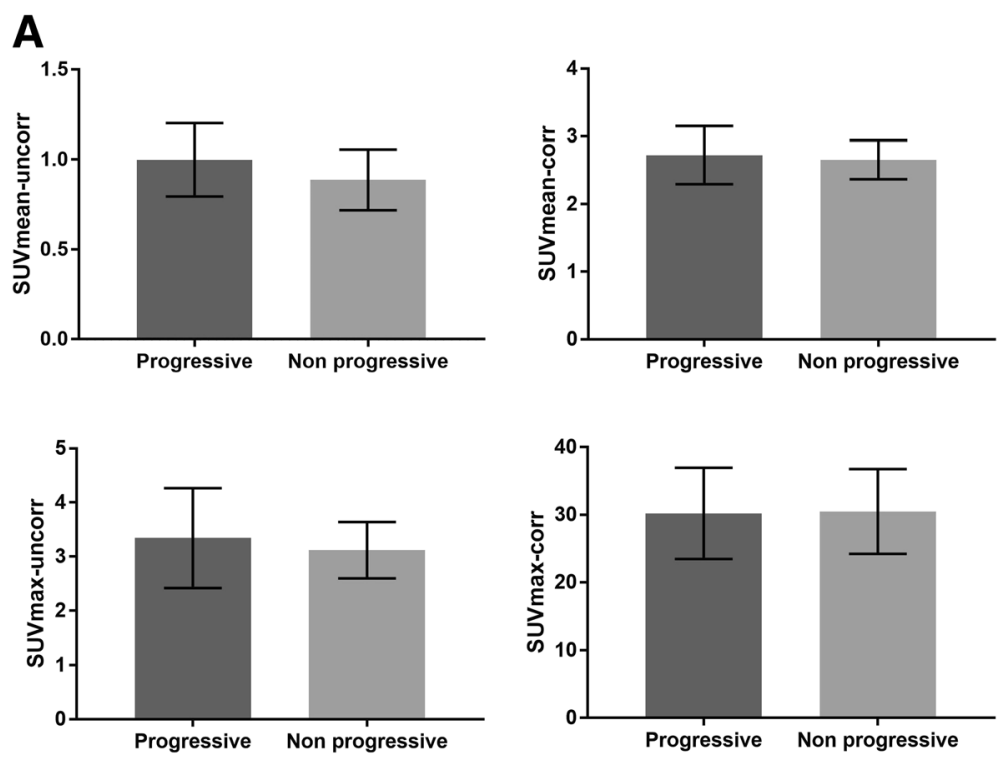

B
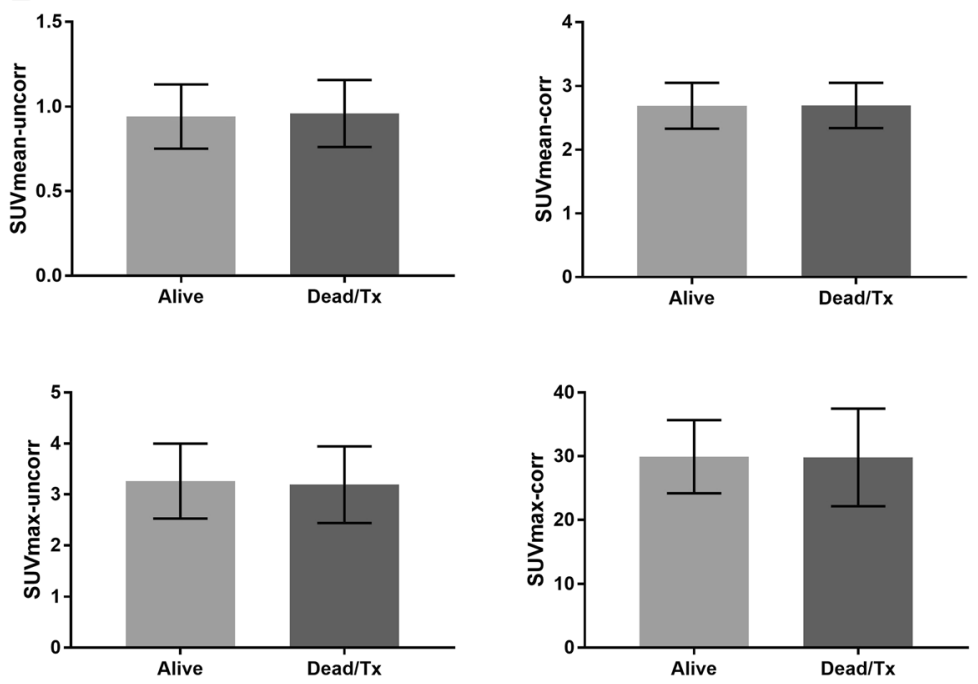

Fig. 5 Comparison between PET/CT values corrected or not for the density and the clinical evolution. Patients were classified according to progressive $(n=15)$ or non-progressive $(n=14)$ disease $(\mathbf{a})$ or to their vital status at the end of the study (alive $(n=19)$ vs death or lung transplanted $(n=12))(\mathbf{b})$. No significant difference was noticed in all comparisons

for lung density thus eliminates correlations between SUVmean and respiratory function variables and reduces the influence of changes in volumes on the metabolic measurements. The normalized measurements are then more directly related to cellular and tissue changes in the lung parenchyma itself. Accordingly, SUVmean-corr was correlated with the DLCO, and the significance of this correlation was supported by the correlation with the desaturation post-exercise during the 6MWT. In other words, increase glucose metabolism estimated by SUVmean-corr indicates a reduction in gas diffusion in the lungs and an increase in oxygen desaturation of the blood after exercise. The study therefore suggests a link between the pulmonary tissue glucose uptake and the status of the alveolar-capillary barrier. In IPF, there is a proliferation of fibroblasts (especially in fibroblastic foci), covered by 
activated epithelial cells (Wolters et al., 2014). Activated fibroblasts have an increased metabolic activity with increased glucose consumption and glycolysis (Xie et al., 2015). Studies also revealed an increased expression of the glucose transporter 1 (GLUT-1) in fibroblasts from IPF patients as well as in epithelial cells and inflammatory cells (El-Chemaly et al., 2013; Cho et al., 2017; Andrianifahanana et al., 2016). Interestingly, GLUT-1 is the main transporter of glucose and [18F]-FDG (El-Chemaly et al., 2013). The increase in SUVmean-corr could therefore be attributed to activated fibroblasts, epithelial cells, or possibly some inflammatory cells, either by an increase in metabolic activity per cell, or by an increase in their number per dense tissue volume, or by a combination of both phenomena. The impact of fibroblastic activity on [18F]-FDG uptake in IPF is supported by a preclinical study on a mouse model of pulmonary fibrosis. In this study, Bondue et al. demonstrated [18F]-FDG lung uptake at the late fibrotic stage of the model, whereas labeled leukocyte recruitment is greatly reduced at this stage (Bondue et al., 2015). The limited influence of the inflammatory cells on the [18F]-FDG uptake in IPF is confirmed in the present study by the lack of correlation between the SUV values and the BAL cytology, in line with similar results obtained by Justet and colleagues (Justet et al., 2017).

Altogether PET/CT evaluation in IPF could provide some additional information about disease activity and prognosis. This is supported by a recent publication showing that adding uncorrected PET parameters to the GAP stage (PET-modified GAP staging) increases the predictive power of the GAP classification (Win et al., 2018). In our study, we demonstrate that the SUVmean-corr is also higher in GAP stage 3. This could indicate that a higher SUVmean-corr is associated with a higher mortality risk. Nevertheless, only 5 patients were classified in stage 3. Moreover, there is a relatively important overlap of the values between GAP stages without a clear threshold above which patients could be clearly differentiated among these stages. Therefore, further studies are needed to determine the extent to which PET data can improve the predictive value of the GAP classification.

Our study presents several limitations: it is a single-center study based on a relatively small number of patients. Also, the follow-up period was limited to 1 year. Finally, most patients received an antifibrotic treatment (pirfenidone or nintedanib) during the follow-up period, which may change their clinical evolution and influence correlations with baseline PET/CT values. These limitations could perhaps explain the lack of correlation found between SUV values (corrected or uncorrected for lung density) and patients' functional and clinical evolution at 1 year.

\section{Conclusions}

In conclusion, the estimated [18F]-FDG uptake with no correction for lung density is tightly related to the lung volumes measurements in IPF; this relation is strongly influenced by the lung density since it disappears when a correction for lung density is applied. To evaluate the intrinsic activity of the disease with [18F]-FDG at the pulmonary tissue level, this correction appears necessary. This correction reveals a relationship between [18F]-FDG uptake in the pulmonary tissue and gas transfer across the disturbed alveolar-capillary barrier in IPF. Despite a relationship with the GAP classification, we found no correlation between [18F]-FDG uptake and most indices of clinical evolution at 1 year. Further studies in larger populations and with a longer follow-up are warranted to establish the prognostic value of corrected SUV values in IPF. 


\section{Additional files}

Additional file 1: Table S1. correlation DLCO, lung volumes, cellularity of the BAL, walk distance at 1 year and all PET parameters measured at baseline. (DOCX $25 \mathrm{~kb}$ )

Additional file 2: Figure S1. Correlation between the SUVmax-corr and GAP index. (JPG 57 kb)

Additional file 3: Figure S2. Correlation SUVmean-corr and delta $\mathrm{SaO}_{2}$ post exercise between baseline value and after 1 year of follow up. (JPG $73 \mathrm{~kb}$ )

\section{Abbreviations}

18F-FDG: 2-deoxy-2-(18F)fluoro-D-glucose, or fluorodeoxyglucose; 6MWT: 6-min walking test; BAL: Bronchoalveolar lavage; DLCO: Diffusion capacity of the lungs for carbon monoxide (CO); FVC: Forced vital capacity; GAP index: A score based on gender (G), age (A), and physiological variables (P); GLUT-1: Glucose transporter 1; HRCT: High-resolution computed tomography; HU: Hounsfield units; ILD: Interstitial lung diseases; IPF: Idiopathic Pulmonary Fibrosis; NS: Not significant; PET/CT: Positron emission tomography with computed tomography; PFT: Pulmonary function tests; $\mathrm{SaO}_{2}$ : Oxygen saturation; SD: Standard deviation; SUV: Standardized uptake value; SUVmean-corr; SUVmax-corr: SUV corrected for lung density; SUVmean-uncorr; SUVmax-uncorr: SUV uncorrected for lung density; TLC: Total lung capacity; VC: Vital capacity

\section{Acknowledgements}

The publication of this article was supported by funds of the European Association of Nuclear Medicine (EANM).

Funding

None.

\section{Availability of data and materials}

The datasets used and/or analysed during the current study are available from the corresponding author on reasonable request.

\section{Authors' contributions}

AC collected, analyzed and interpreted the patient data, performed statistical analyses and helped draft the manuscript. GVS collected, analyzed and interpreted all PET data, and helped draft the manuscript. SG contributed to the design of the study, assisted in the analysis and interpretation of the data, and helped draft the manuscript. BB contributed to the design of the study, assisted in the analysis and interpretation of the data, selected the patients, performed patient follow-up and helped draft the manuscript. All authors read and approved the final manuscript.

\section{Ethics approval and consent to participate}

All procedures performed in this study involving human participants were in accordance with the Erasme hospital Ethics Committee (ref. P2016/427; EudraCT/CCB: NA), and with the 1964 Helsinki declaration and its later amendments or comparable ethical standards.

\section{Consent for publication}

Informed consent was obtained from all individual participants included in the study.

\section{Competing interests}

The authors declare that they have no competing interests.

\section{Publisher's Note}

Springer Nature remains neutral with regard to jurisdictional claims in published maps and institutional affiliations.

Received: 5 July 2018 Accepted: 7 October 2018

Published online: 14 November 2018

\section{References}

Andrianifahanana M, Hernandez DM, Yin X, Kang J-H, Jung M-Y, Wang Y et al (2016) Profibrotic up-regulation of glucose transporter 1 by TGF- $\beta$ involves activation of MEK and mammalian target of rapamycin complex 2 pathways. FASEB 30(11):3733-3744

Archontogeorgis K, Steiropoulos P, Tzouvelekis A, Nena E, Bouros D (2012) Lung cancer and interstitial lung diseases: a systematic review. Pulm Med 2012:315918

Best AC, Lynch AM, Bozic CM, Miller D, Grunwald GK, Lynch DA (2003) Quantitative CT indexes in idiopathic pulmonary fibrosis: relationship with physiologic impairment. Radiology 228(2):407-414

Bondue B, Sherer F, Van Simaeys G, Doumont G, Egrise D, Yakoub Y et al (2015) PET/CT with 18F-FDG- and 18F-FBEM-labeled leukocytes for metabolic activity and leukocyte recruitment monitoring in a mouse model of pulmonary fibrosis. J Nucl Med 56(1):127-132

Cho SJ, Moon J-S, Lee C-M, Choi AMK, Stout-Delgado HW (2017) Glucose transporter 1-dependent glycolysis is increased during aging-related lung fibrosis, and Phloretin inhibits lung fibrosis. Am J Respir Cell Mol Biol 56(4):521-531

Collard HR, Ryerson CJ, Corte TJ, Jenkins G, Kondoh Y, Lederer DJ et al (2016) Acute exacerbation of idiopathic pulmonary fibrosis. An international working group report. Am J Respir Crit Care Med 194(3):265-275 
El-Chemaly S, Malide D, Yao J, Nathan SD, Rosas IO, Gahl WA et al (2013) Glucose Transporter-1 distribution in fibrotic lung disease. Chest 143(6):1685-1691

Groves AM, Win T, Screaton NJ, Berovic M, Endozo R, Booth $\mathrm{H}$ et al (2009) Idiopathic pulmonary fibrosis and diffuse parenchymal lung disease: implications from initial experience with 18F-FDG PET/CT. J Nucl Med 50(4):538-545

Holman BF, Cuplov V, Millner L, Hutton BF, Maher TM, Groves AM et al (2015) Improved correction for the tissue fraction effect in lung PET/CT imaging. Phys Med Biol 60(18):7387-7402

Justet A, Laurent-Bellue A, Thabut G, Dieudonné A, Debray M-P, Borie R, et al. [(18)F]FDG PET/CT predicts progression-free survival in patients with idiopathic pulmonary fibrosis. Respir Res 2017;18(1):74

Lambrou T, Groves AM, Erlandsson K, Screaton N, Endozo R, Win T et al (2011) The importance of correction for tissue fraction effects in lung PET: preliminary findings. Eur J Nucl Med Mol Imaging 38(12):2238-2246

Lee EYP, Wong CS, Fung SL, Yan PK, Ho JCM (2014) SUV as an adjunct in evaluating disease activity in idiopathic pulmonary fibrosis - a pilot study. Nucl Med Commun 35(6):631-637

Ley B, Ryerson CJ, Vittinghoff E, Ryu JH, Tomassetti S, Lee JS et al (2012) A multidimensional index and staging system for idiopathic pulmonary fibrosis. Ann Intern Med 156(10):684-691

Meissner H-H, Soo Hoo GW, Khonsary SA, Mandelkern M, Brown CV, Santiago SM (2006) Idiopathic pulmonary fibrosis: evaluation with positron emission tomography. Respir Int Rev Thorac Dis 73(2):197-202

Nobashi T, Kubo T, Nakamoto Y, Handa T, Koyasu S, Ishimori T et al (2016) 18F-FDG uptake in less affected lung field provides prognostic stratification in patients with interstitial lung disease. J Nucl Med 57(12):1899-1904

Ohkubo H, Kanemitsu Y, Uemura T, Takakuwa O, Takemura M, Maeno K et al (2016) Normal lung quantification in usual interstitial pneumonia pattern: the impact of threshold-based volumetric CT analysis for the staging of idiopathic pulmonary fibrosis. PLoS One 11(3):e0152505

Raghu G, Collard HR, Egan JJ, Martinez FJ, Behr J, Brown KK et al (2011) An official ATS/ERS/JRS/ALAT statement: idiopathic pulmonary fibrosis: evidence-based guidelines for diagnosis and management. Am J Respir Crit Care Med 183(6):788-824

Salisbury ML, Xia M, Zhou Y, Murray S, Tayob N, Brown KK et al (2016) Idiopathic pulmonary fibrosis: gender-age-physiology index stage for predicting future lung function decline. Chest 149(2):491-498

Spagnolo P, Rossi G, Cavazza A (2014) Pathogenesis of idiopathic pulmonary fibrosis and its clinical implications. Expert Rev Clin Immunol 10(8):1005-1017

Umeda Y, Demura Y, Morikawa M, Anzai M, Kadowaki M, Ameshima S et al (2015) Prognostic value of dual-time-point 18FFDG PET for idiopathic pulmonary fibrosis. J Nucl Med 56(12):1869-1875

Win T, Screaton NJ, Porter JC, Ganeshan B, Maher TM, Fraioli F et al (2018) Pulmonary 18F-FDG uptake helps refine current risk stratification in idiopathic pulmonary fibrosis (IPF). Eur J Nucl Med Mol Imaging 45(5):806-815

Win T, Thomas BA, Lambrou T, Hutton BF, Screaton NJ, Porter JC et al (2014) Areas of normal pulmonary parenchyma on HRCT exhibit increased FDG PET signal in IPF patients. Eur J Nucl Med Mol Imaging 41(2):337-342

Wolters PJ, Collard HR, Jones KD (2014) Pathogenesis of idiopathic pulmonary fibrosis. Annu Rev Pathol 9:157-179

Xie N, Tan Z, Banerjee S, Cui H, Ge J, Liu R-M et al (2015) Glycolytic reprogramming in Myofibroblast differentiation and lung fibrosis. Am J Respir Crit Care Med 192(12):1462-1474

\section{Submit your manuscript to a SpringerOpen ${ }^{\circ}$ journal and benefit from:}

- Convenient online submission

- Rigorous peer review

- Open access: articles freely available online

- High visibility within the field

- Retaining the copyright to your article

Submit your next manuscript at $>$ springeropen.com 